\title{
RNA helicase DDX5 enables STAT1 mRNA translation and interferon signaling in hepatitis B virus replicating hepatocytes.
}

\author{
Jiazeng Sun ${ }^{1,3}$, Guanhui $\mathrm{Wu}^{2,3}$, Wen-Hung Wang ${ }^{4}$, Zhengtao Zhang ${ }^{5}$, Philippe Merle ${ }^{6}$, \\ Lijian Hui ${ }^{5}$, Danzhou Yang ${ }^{2,3}$ and Ourania Andrisani ${ }^{1,3^{*}}$
}

${ }^{1}$ Department of Basic Medical Sciences, ${ }^{2}$ Department of Medicinal Chemistry and Molecular Pharmacology, ${ }^{3}$ Purdue Center for Cancer Research, and ${ }^{4}$ Gene Editing Core, Bindley Biosciences Center, Purdue University, West Lafayette, IN 47907. ${ }^{5}$ Shanghai Institute of Biochemistry and Cell Biology, Chinese Academy of Sciences, Suzhou, Jiangsu 215121, China. ${ }^{6}$ Department of Hepatology, Hôpital de la Croix-Rousse, Hospices Civils de Lyon, Université Lyon 1, Lyon, France

*corresponding author: andrisao@ purdue.edu

Department of Basic Medical Sciences, Purdue University 201 S. University Street

West Lafayette, IN 47907-2064

Phone: 765-494-8131

Keywords: RNA helicase DEAD box protein 5 (DDX5), STAT1, G-quadruplex, Interferon Signaling, Hepatitis B Virus, Hepatocellular Carcinoma

Conflict of Interest: Nothing to disclose/No conflict

Financial support: This work was supported by NIH grants DK044533 to OA and CA177585 to DY. Shared Resources (Genomics and Bioinformatics Facilities) supported by NIH grant P30CA023168 to Purdue Center for Cancer Research, and NIH/NCRR RR025761.

Word count: 4,150 words excluding references and figure legends; Figures: 8 


\section{ABSTRACT}

Objective: RNA helicase DDX5 is downregulated during hepatitis B virus (HBV) replication, and poor prognosis HBV-related hepatocellular carcinoma (HCC). The objective of this study is to understand the significance of DDX5 downregulation during HBV infection and poor prognosis HCC, as it pertains to therapy of chronically HBV-infected patients with HCC. IFN- $\alpha$ therapy is effective only for a subgroup of HBV-infected patients, for reasons not understood. Herein, we investigated a novel mechanism of STAT1 translational control, involving DDX5mediated resolution of a G-quadruplex structure, located at the 5'UTR of STAT1 mRNA, enabling STAT1 translation.

Design and Results: Molecular, pharmacologic, and biophysical assays were used together with cellular model of HBV replication, HCC cell lines, and HBV-related liver tumors. We observed, the protein level of DDX5 correlated with that of transcription factor STAT1. We show, DDX5 regulates STAT1 mRNA translation by resolving a G-quadruplex (rG4) RNA structure, proximal to the 5' end of STAT1 5'UTR. We employed luciferase reporter assays comparing wild type (WT) vs. mutant (MT) rG4 sequence, rG4-stabilizing compounds, CRISPR/Cas9 editing of the rG4 sequence, and circular dichroism determination of rG4 structure. Ribonucleoprotein assays identified direct DDX5 binding to WT but not MT rG4. Importantly, liver cancer cell lines expressing low DDX5 exhibited reduced interferon response, while immunohistochemistry of HBV-related HCCs exhibiting absence of DDX5, also lacked STAT1. Conclusion: DDX5 resolves a G-quadruplex structure in 5'UTR of STAT1 mRNA, enabling STAT1 translation. DDX5 is a key regulator of the dynamic range of interferon response during innate immunity and adjuvant IFN- $\alpha$ therapy. 


\section{Introduction}

Interferon Type I/III signaling acting through the JAK/STAT pathway exerts antiviral, antitumor, and immunomodulatory effects $(1,2)$. Effects of IFN- $\alpha$ and IFN- $\beta$ are mediated by tyrosine phosphorylation of transcription factors STAT1 and STAT2, followed by formation of ISGF3 complex via interaction with IRF9, translocation to the nucleus, and transcriptional induction of Interferon Stimulated Genes (ISGs). In addition to tyrosine phosphorylation, STAT1 activity is further modulated by modifications including methylation (3), acetylation (4) and ubiquitination (5). Since STAT1 is involved in all types (I/III) of interferon signaling (6), these post-translational modifications influence all types (I/III) of interferon signaling, depending on cellular context. Herein, we provide evidence for a novel mechanism of STAT1 regulation, involving translational control of STAT1 mRNA, studied in the context of viral replication in hepatocytes harboring the hepatitis B virus (HBV).

Hepatitis B virus (HBV) infection is a significant global health problem with more than 250 million chronically infected patients. Chronic HBV infection is associated with progressive liver disease, cirrhosis, and development of hepatocellular carcinoma (HCC). Curative treatments for early stage HCC include liver resection, transplantation, or local ablation. In advanced stage HCC, multi-kinase inhibitors (7-9), offer only palliative benefits. In addition, because IFNs exert both antiviral and anti-proliferative effects $(10,11)$, adjuvant IFN- $\alpha$ therapy has been extensively applied for treatment of chronically HBV-infected patients with HCC. Unfortunately, many patients do not respond to IFN- $\alpha$ (12). Importantly, HCC patients with increased expression of the ISG IFIT3, a direct transcription target of STAT1 (13), predict positive response to IFN- $\alpha$ therapy (14), suggesting IFN- $\alpha$ non-responders lack STAT1 activation and/or expression. 
Viruses have evolved effective strategies to hijack cellular pathways for their growth advantage, including mechanisms for immune evasion. In our studies, we have identified a cellular mechanism hijacked by HBV associated with both viral biosynthesis and poor prognosis HBV-related HCC (15-17). This mechanism involves the chromatin modifying PRC2 complex and RNA helicase DDX5. PRC2 mediates repressive histone modifications (H3K27me3)(18), while RNA helicase DDX5 is involved in transcription, epigenetic regulation, miRNA processing, mRNA splicing, decay, and translation $(19,20)$. Interestingly, HBV infection downregulates DDX5 via induction of two microRNA clusters (21), miR17 92 and miR106 25 (15-17). Moreover, HBV replicating cells with reduced DDX5 exhibit Wnt activation, resistance to chemotherapeutic agents (21), and reduced protein levels of STAT1, as we describe herein.

In this study, we provide evidence that DDX5 exerts additional functions in HBV infected cells, i.e., modulation of the innate immune response, via a novel mechanism of translational control of STAT1. Specifically, RNA helicase DDX5 resolves a secondary RNA structure called G-quadruplex (22), located in the 5' untranslated region (5'UTR) of STAT1 mRNA, thereby enabling its translation. G-quadruplexes are four-stranded structures formed in guanine (G)-rich sequences (23), and when located in 5'UTRs of mRNAs, influence posttranscriptional regulation of gene expression (20). One of the first examples of translational repression by an RNA G-quadruplex (rG4) was demonstrated for human NRAS mRNA (24). Bioinformatics analysis estimated nearly 3,000 5'UTR rG4s in the human genome (24), and rG4-sequencing of polyA+ HeLa RNA generated a global map of thousands rG4 structures (25). Recently, it was discovered that DDX5 proficiently resolves both RNA and DNA G4 structures (26). 
The significance of this mechanism of translational control of STAT1 is dependence on the protein level and activity of the rG4-resolving helicase. In terms of IFN (I/III) signaling and the innate response of $\mathrm{HBV}$ infected cells, our results presented herein demonstrate that the magnitude of the interferon response is influenced by this dynamic mechanism of STAT1 translational regulation, dependent on the protein level of DDX5.

\section{Materials and Methods}

Cell culture: Human hepatocellular carcinoma (HCC) cell lines HepG2, Huh7, Snu387, Snu423, HepaRG (27), HepAD38 (28), CLC15 and CLC46 (29) were grown as described, and regularly tested for mycoplasma using PCR Mycoplasma Detection Kit (Abm). HepAD38 cells support HBV replication by tetracycline removal (28). HepAD38 were STR tested by ATCC.

Transfection assays: HepAD38, Huh7, and HepaRG cells $\left(0.3 \times 10^{6}\right.$ cells per well of 6 -well plate) were transfected with control (Ctrl) or DDX5 siRNAs (40 nM). Following transfection (48 h), cells were harvested for RNA or protein extraction and analyzed by qRT-PCR or immunoblotting, respectively. HepAD38, Huh7, and HepaRG cells $\left(0.1 \times 10^{6}\right.$ cells per well of 12 well plate) were co-transfected with Renilla luciferase (25 ng) and WT STAT1-5'UTRLuciferase (pFL-SV40-STAT1-5'UTR a gift from Dr. Ming-Chih Lai) (50 ng) or mutant MTSTAT1-5'UTR-Luciferase (50 ng) using Lipofectamine 3000 (Life Technologies). In HepAD38 cells, HBV replication was induced by tetracycline removal 4 days prior to transfection with Renilla and Firefly luciferase vectors. Ctrl or DDX5 siRNAs (40 nM each) were co-transfected with Renilla and Firefly luciferase vectors using RNAiMax (Life Technologies). Plasmids used are listed in Supporting Table S1. Firefly luciferase activity was measured $24 \mathrm{~h}$ after transfection 
using Dual Luciferase Assay system (Promega) according to manufacturer's protocol, normalized to Renilla luciferase.

RNA preparation and qRT-PCR assay: RNA preparation and qRT-PCR performed according to manufacturer's instructions, employing commercially available kits (Supporting Table S2). Primer sequences listed in Supporting Table S3.

Immunoblot and Immunohistochemistry assays performed as described (16). Densitometric analysis of immunoblots was by ImageJ. Antibodies used are listed in Supporting Table S4.

Ribonucleoprotein Immunoprecipitation (RIP) Assay: RIP assays employed the Magna RIPTM RNA-Binding Protein Immunoprecipitation Kit (Millipore Sigma) following manufacturer's instructions. Primer sequences and antibodies used are listed in Supporting Table $\mathrm{S} 3$ and $\mathrm{S} 4$, respectively.

RNA pull down assay: RNA folding and pull down assays were performed as described with modifications (30). Briefly, synthetic 5'-biotinylated rG4 (Bio-rG4) and rG4mut (Bio-rG4mut) RNA oligonucleotides (Millipore Sigma) were diluted to $5 \mathrm{mM}$ in folding buffer, heated to 95 ${ }^{\circ} \mathrm{C}$, and cooled to $25^{\circ} \mathrm{C}$. G-quadruplex formation determined by circular dichroism (CD), as described below. Whole cell extracts from HepAD38, Huh7, and HepaRG cells were incubated with folded biotinylated RNAs (Table S3), followed by pull down with streptavidin beads (Promega). Bound proteins analyzed by immunoblotting.

Circular Dichroism spectroscopy of RNA oligonucleotides performed as described (31), employing Jasco J-1100 spectropolarimeter equipped with thermoelectrically controlled cell holder. CD measurements were performed using quartz cell with optical path length of $1 \mathrm{~mm}$. Blank sample contained only buffer (25mM Tris-HCL, pH 7.4). Each CD spectroscopy 
measurement was the average of two scans, collected between 340 and $200 \mathrm{~nm}$ at $25^{\circ} \mathrm{C}$, scanning speed $50 \mathrm{~nm} / \mathrm{min}$. CD melting experiments were performed at $264 \mathrm{~nm}$ with heating rate of $2^{\circ} \mathrm{C} / \mathrm{min}$ between $25^{\circ} \mathrm{C}$ and $95^{\circ} \mathrm{C}$. RNA sample concentration was $10 \mu \mathrm{M}$.

CRISPR/Cas9 gene editing: Huh7 and HepaRG cells were used to introduce indels targeting essential nucleotides of the G-quadruplex structure in 5' UTR region of DDX5 gene (32). Ribonucleoprotein (RNP) of Cas9-2NLS (10 pmol) and guide RNA (50 pmol, Synthego) were loaded onto a $10 \mu \mathrm{l}$ Neon Tip, and electroporated into $1 \times 10^{5}$ Huh7 and HepaRG cells, using Neon Transfection System at $1200 \mathrm{~V}$, for $20 \mathrm{msec}$ and 4 pulses (ThermoFisher Scientific), according to manufacturer's instructions. Genomic DNA was isolated and used for rapid PAGE genotyping (33) to validate incorporation of indels, $48 \mathrm{~h}$ after electroporation. Validated pools of cells were subjected to clonal selection. Isolated single colonies were confirmed by rapid PAGE genotyping and allelic sequencing.

Statistical Analysis: Statistical analysis was performed using unpaired $t$ test in GraphPad Prism version 6.0 (GraphPad Software, San Diego, CA). Differences were considered significant when $p<0.05$.

\section{Results}

We examined various liver cancer cell lines, HepG2, Huh7, Snu423 and HepaRG for IFN- $\alpha$ response, employing immunoblots for activated phospho-STAT1 (T701) and expression of ISG IRF9. All cell lines responded to IFN- $\alpha$, including the HBV replicating HepAD38 cell line that contains a stably integrated copy of the HBV genome under control of the Tet-off promoter (28) (Figure 1A and Supplementary Figure S1A-B). In the absence of IFN- $\alpha$ treatment, HBV replication in HepAD38 cells exerted a small but reproducible reduction on STAT1 protein level 
(Figure 1A). Following IFN- $\alpha$ treatment for 24 h, levels of STAT1, p-STAT1, and downstream ISGs IRF9 and IFTM3 were similarly reduced (Figure 1A and Supplementary Figure S1C). Likewise, HBV replication exerted a reproducible decrease in protein level of DDX5, irrespective of IFN- $\alpha$ treatment (Figure 1A), as reported previously (16).

To determine whether DDX5 downregulation was associated with downregulation of STAT1 observed during HBV replication (Figure 1A), we transfected increasing amounts of siRNA for DDX5 in HepAD38 cells. Surprisingly, reduction in DDX5 protein resulted in progressive reduction in STAT1 protein level (Figure 1B and Supplementary Figure S2). DDX5 knockdown in Huh7 and HepaRG cell lines also resulted in reduced STAT1 protein (Figure 1B and Supplementary Figure S2), whose t1/2 was quantified to be 16 h (Supplementary Figure S3A-B). Next, we examined STAT1 protein levels in three clonal DDX5-knockdown cell lines, KD2, KD3 and KD5, constructed in HepAD38 cells. These cell lines lacked STAT1 protein (Figure 1C) and I IFN- $\alpha$ response (Supplementary Figure S3C). Interestingly, STAT1 mRNA levels were unaffected in HBV replicating HepAD38 cells (Figure 1D), in DDX5 knockdown HepAD38 cells (Figure 1E), and upon transient siRNA-mediated DDX5 knockdown in HepAD38, Huh7, and HepaRG cells (Figure 1F), thereby excluding DDX5 effects on STAT1 transcription.

The STAT1 gene contains 24 introns. Accordingly, we examined whether intron detention or aberrant splicing are regulated by DDX5. qRT-PCR and RNA-seq intron data analyses of STAT1 in WT HepAD38 cells vs. DDX5-knockdown cells excluded STAT1 intron detention (Supplementary Figure S4A). Likewise, comparison of STAT1 mRNA sequence from WT vs. DDX5-knockdown cells, excluded aberrant splicing involving the mRNA splice site in 
proximity to AUG (Supplementary Figure S4B). These results suggested DDX5 regulates STAT1 mRNA translation.

\section{rG4 structures in 5'UTR of human STAT1 mRNA}

Having excluded DDX5 effects on STAT1 mRNA transcription and processing, we reasoned, the information for post-transcriptional regulation must be located in the sequence or structure of STAT1 5' UTR. Surprisingly, the rG4-seq transcriptomic studies by Kwok et al (25) identified the 5'UTR of human STAT1 mRNA as a high probability mRNA harboring rG4 structures (Supplementary Figure S4C). To test this hypothesis, and using NRAS as our positive control (24), we examined the effect of several G4 stabilizing compounds (Figure 2A), including PhenDC3 and RR82 (22), on STAT1 protein level. Both compounds reduced STAT1 and NRAS protein level, without affecting STAT1 mRNA (Figure 2B). Similarly, we tested the effect of the G4-interactive TMPyP4 and its corresponding non-G4-interactive TMPyP2 compound (34) on STAT1 protein and mRNA levels, using HepAD38 and Huh7 cells (Figure 2C). The G4interactive TMPyP4, similar to RR82, suppressed STAT1 protein levels, whereas the non-G4 interactive TMPyP2 exerted no effect. Importantly, these compounds did not affect STAT1 mRNA levels (Figure 2C). We interpret these results to mean the 5'UTR of STAT1 contains a putative rG4 structure, regulating STAT1 expression post-transcriptionally.

Employing an expression vector driven by the SV40 promoter, we cloned nucleotides (nt) +1 to +400 of the 5'UTR of STAT1 upstream of Firefly (F.) luciferase gene. This 5'UTR region contains three putative rG4s, labeled as rG4-1, rG4-2 and rG4-3 (Supplementary Figure S4C). Importantly, rG4-1 is located 30 nt downstream from the start of the 5'UTR. Earlier studies demonstrated rG4s situated proximal or within the first 50 nt to the 5 ' end of the 5'UTR are 
functional and effective in repressing translation (35). Based on this reasoning, we focused our analysis on rG4-1 and constructed a mutant (MT) rG4-1 (Figure 3A), with G to A substitutions within the putative rG4-1 sequence. Transfection in HepAD38 cells of expression vectors containing WT and MT rG4-1 demonstrated a statistically significant increased F. luciferase activity from MT rG4-1 in comparison to WT rG4-1 vector, while no changes were observed at the mRNA level, both in HepAD38 and Huh7 cells (Figure 3B). Mutational analyses of rG4-2 and rG4-3 sequences excluded a similar role on STAT1 expression (Supplementary Figure S5A and B). Next, we examined the effect of HBV replication, using HepAD38 cells, on F. luciferase activity expressed from WT and MT rG4-1 vectors. HBV replication reduced F. luciferase activity only from the WT rG4-1 containing vector, without an effect on F. luciferase mRNA expression (Figure 3C). Similar results were observed by siRNA knockdown of DDX5 (siDDX5) (Figure 3C). Employing these expression vectors, we also tested the effect of the G4stabilizing compounds RR82 and TMPyP4. Both drugs reduced expression only from the WT rG4-1 vector, without an effect on mRNA levels of F. luciferase, tested in HepAD38 (Figure 3D) and Huh7 (Figure 3C) cells. Taken together, these results identify the rG4-1 sequence as a functional element in 5'UTR of STAT1 mRNA, regulating its translation.

\section{Genomic editing of rG4-1 increases STAT1 protein levels}

Using clustered regularly interspaced short palindromic repeats (CRISPR)/Cas9 technology (36) we edited the genomic rG4-1 sequence of STAT1 in Huh7 and HepaRG cell lines. Several clones were isolated and sequenced, and protein and mRNA levels of STAT1 were determined (Figure 4 and Supplementary Figure S6). DNA sequencing of HepaRG clones C5 and C9 (Supplementary Figure S6) demonstrated C5 cells contain rG4-1 deletions in both alleles, while C9 cells have rG4 changes only in one allele (Figure 4B). All clones analyzed from Huh7 and 
HepaRG cells exhibited statistically significant and reproducible increases in protein level of STAT1 in comparison to WT (unedited) cells, while STAT1 mRNA levels remained unchanged (Figure 4). These results are also supported by luciferase reporter assays containing the edited rG4-1 sequences (Supplementary Figure S7).

Interestingly, DDX5 knockdown by transfection of DDX5 siRNA had no effect on STAT1 protein levels of clone C5, containing edited rG4 sequence on both STAT1 alleles (Figure 5A). By contrast, siDDX5 reduced STAT1 protein levels in WT and C9 cells (Figure 5A). Likewise, STAT1 protein levels of clone C5 were resistant to G4-stabilizing drugs RR82 and TMPyP4, while these compounds reduced STAT1 levels in both WT and C9 cells (Figure 5B). Next, we examined the interferon response of WT, C5, and C9 cells as a function of cotreatment with RR82 (Figure 5C). In contrast to WT and C9 cells, the IFN- $\alpha$ response of C5 cells was not inhibited by RR82, in terms of STAT1 protein level and activation, as well as induction of IRF9 and IFITM3 (Figure 5C and Supplementary Figure S8). These results support that both alleles of clone C5 contain nonfunctional rG4-1 structures in 5'UTR of STAT1 mRNA.

\section{The rG4-1 sequence of STAT1 forms G-quadruplex in vitro.}

To directly determine whether the rG4-1 sequence in 5'UTR of STAT1 indeed forms a Gquadruplex structure, we employed CD spectroscopy, a method that enables study of nucleic acid secondary structure $(31,37)$. RNA oligonucleotides were synthesized (Figure 6A) that corresponded to the indicated sequences of rG4-1 found in WT STAT1 mRNA, and in each allele of clones C5 and C9. Since G4 oligonucleotides can form higher-order structures, we included U-streches at the 5' and 3' of the indicated RNA oligonucleotides, and confirmed these RNA molecules were in monomeric form, employing native PAGE (Supplementary Figure S9). 
The CD spectrum and thermal stability of these RNA oligonucleotides were studied as a function of $\mathrm{K}+$ addition $(100 \mathrm{mM})$, a cation that stabilizes $\mathrm{G} 4$ structures (38). The melting temperature (Tm) of each RNA oligonucleotide is shown (Figure 6D). Addition of $100 \mathrm{mM} \mathrm{KCl}$ increased the Tm of the WT, C9-1, and C9-2 RNA oligonucleotides, supporting formation of stable rG4 structures. By contrast, oligonucleotide C5-1 and C5-2 exhibited no or very small Tm increase in comparison to WT. These results indicate, the WT, C9-1 and C9-2 sequences form stable rG4 structures, whereas C5-1 and C5-2 lack formation of this structure. These biophysical results are congruent with the biological data presented herein, and demonstrate the role of rG4-1 structure in STAT1 translational control.

\section{DDX5 selectively binds STAT1 mRNA with WT rG4-1}

To determine whether DDX5 interacts directly with STAT1 mRNA, we performed ribonucleoprotein immunoprecipitation (RIP) assays employing DDX5 antibody and IgG as negative control. In HepAD38 and Huh7 cells endogenous, immunoprecipitated DDX5 was found in association with STAT1 mRNA, while IgG did not exhibit such association (Figure 7A and B). Next, we performed RIP assays in HepaRG cells, WT and clones C5 and C9. Interestingly, endogenous DDX5 associated with STAT1 mRNA in WT and clone C9 cells, while STAT1 mRNA expressed in clone C5, containing mutated rG4-1 sequence on both alleles, lacked binding to DDX5 (Figure 7C).

To further confirm that DDX5 binds the rG4-1 sequence, biotinylated oligonucleotides containing WT or mutant rG4-1 (Figure 7D) were incubated in $100 \mathrm{mM} \mathrm{K}+$ that stabilizes the G4 structure or $100 \mathrm{mM} \mathrm{Li}+$ that does not (38). Mutant rG4-1 does not form stable G4 structure even

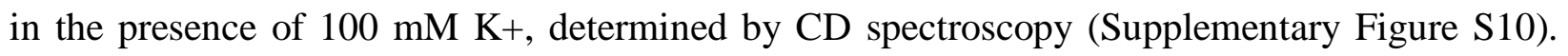


Next, pre-folded RNA oligonucleotides incubated with lysates from HepAD38, Huh7, and HepaRG cells were bound to streptavidin beads, and the retained proteins analyzed by DDX5 immunoblots. Indeed, these pull-down assays show DDX5 selectively binds the WT rG4-1 whose structure is stabilized by $100 \mathrm{mM} \mathrm{K+(Figure} \mathrm{7D).}$

\section{STAT1 expression in liver cancer cell lines and HBV-related liver tumors}

To further establish the biological connection between DDX5 and STAT1 protein levels relative to interferon response, we quantified IFN- $\alpha$ response in liver cancer cell lines exhibiting different levels of DDX5 protein. We compared cell lines Snu387 vs. Snu423, and CLC15 vs. CLC46 (29), by performing immunoblots of lysates treated with increasing amount of IFN- $\alpha$ for $12 \mathrm{~h}$ (Figure 8A and Supplementary Figure S11). Quantification of the immunoblots shows the pSTAT1, STAT1, DDX5 and IRF9 signal normalized to the baseline p-STAT1 signal obtained in the absence of IFN- $\alpha$ (Figure 8B). The results demonstrate, low level of DDX5 correlates with reduced STAT1 protein, STAT1 activation, and response to IFN- $\alpha$. Likewise, we quantified the STAT1 mRNA from each cell line as a function of IFN- $\alpha$, and normalized STAT1 protein levels quantified from the immunoblots (Figure 8A) relative to STAT1 mRNA. In comparison to Snu387 cells, Snu423 cells that express higher DDX5 protein also exhibit higher STAT1 protein (Figure 8C). Furthermore, we analyzed by immunohistochemistry the expression levels of DDX5 and STAT1 proteins in a small set of HBV-related HCCs. Employing normal human tissues as positive control, we observed absence of immunostaining for both DDX5 and STAT1 in Edmonson's grade 3 HBV-related HCC tumors (Figure 8D), supporting our mechanistic in vitro observations.

\section{Discussion}


Herein, we report the translational control of STAT1, a transcription factor essential for all types (I/III) of interferon signaling, via a G-quadruplex (rG4) structure located at the 5'UTR of STAT1 mRNA. RNA helicase DDX5 resolves this rG4 structure, thereby enabling STAT1 translation. Significantly, this post-transcriptional regulation of STAT1 expression is a cell intrinsic mechanism that influences the dynamic range of interferon response, dependent on protein level and activity of DDX5. The RNA helicase DDX5 regulates every aspect of RNA metabolism (39), and also serves as a barrier to pluripotency (40). Interestingly, it has been noted that pluripotent stem cells are refractory to interferon signaling $(41,42)$, although the mechanism is not yet understood. It is currently unknown whether absence of DDX5 expression or activity contributes to lack of innate immune response in pluripotent stem cells $(41,42)$.

G-quadruplexes (G4) are non-canonical, secondary DNA or RNA structures formed by guanine rich sequences $(22,23,43)$. DNA G4 structures are found in gene promoters, particularly of oncogenes, regulating their transcription (44). Interestingly, recent studies demonstrated that DDX5 resolves both RNA and DNA G4 structures, including a G4 structure found in the MYC promoter, enhancing its transcriptional activity (26). RNA G-quadruplexes (rG4) are primarily located in 5'UTR or 3'UTR of mRNAs, playing important roles in RNA biology, from splicing, stability, mRNA targeting, and translation $(22,43)$. rG4 structures have been identified by various methods in the 5' UTR of NRAS (24), Zic-1(45), Nkx2-5 (46) mRNAs, among other genes $(22,43)$, regulating their translation. Herein, we demonstrate by pharmacologic, molecular, and biophysical approaches the functional significance of the rG4 structure in the 5'UTR of STAT1 mRNA. Specifically, G4-structure stabilizing compounds RR82, PhenDC3 and TMPyP4 reduced the protein level of both STAT1 and NRAS, used as positive control, without affecting STAT1 mRNA level (Figure 2). After excluding other post- 
transcriptional modes of STAT1 regulation, we focused on the rG4-1 sequence located in proximity to the 5' end of STAT1 5'UTR, since it had been identified as a high probability rG4 sequence by the transcriptomic study of Kwok et al (25). Indeed, using an SV40-driven Luciferase reporter, containing the STAT1 5'UTR ( $\mathrm{nt}+1$ to +400) upstream from F. Luciferase, we demonstrated that G4-structure stabilizing compounds suppressed the protein synthesis but not mRNA level of the WT rG4-1 containing luciferase vector (Figure 3). We also edited the genome of Huh7 and HepaRG cells by the CRISPR/Cas9 approach and generated alterations (deletions) of the rG4-1 sequence of STAT1 gene (Figure 4). When both alleles harbored the edited rG4-1 sequence, as in HepaRG clone C5, STAT1 protein levels were increased. Significantly, expression of STAT1 in clone C5 was resistant both to DDX5 knockdown (Figure 5A), and the inhibitory effect of G4-stabilizing compounds (Figure 5B). Circular dichroism and thermal stability measurements directly demonstrated formation of G-quadruplex only by WT and C9 rG4-1 sequences (Figure 6), supporting the biological data. RIP assays using antibody to endogenous DDX5 protein, showed lack of DDX5 binding to STAT1 mRNA from clone C5, containing edited rG4-1 sequence in both alleles. By contrast, DDX5 bound to STAT1 mRNA encoding the WT and C9 rG4-1 sequence (Figure 7). These results were further verified by RNA pulldown assays using synthetic RNA oligonucleotides under $\mathrm{K}+$ conditions enabling formation of the G-quadruplex structure, thereby confirming endogenous DDX5 recognizes the Gquadruplex found at the 5' UTR of STAT1 mRNA (Figure 7). Taken these results together, we conclude, the rG4-1 sequence in 5'UTR of human STAT1 mRNA assumes a G-quadruplex secondary structure. This rG4 structure, stabilized by G4-stabilizing compounds RR82, PhenDC3 and TMPyP4, suppresses STAT1 mRNA translation. RNA helicase DDX5 resolves the rG4 structure, enabling STAT1 mRNA translation. 
Regarding the biological significance of this mechanism, we compared STAT1 levels and the interferon response using two sets of liver cancer cell lines expressing low vs. high DDX5, namely, Snu387 vs. Snu423, and CCL15 and CCL46 derived from HBV-related HCCs (29). We observed, the magnitude of the IFN- $\alpha$ response is a cell intrinsic property, dependent on the DDX5 expression level (Figure 8A-C and Supplementary Figure S11). Furthermore, although DDX5 is an ATPase (39), and arginine methylation by PRMT5 modulates the function of DDX5 in resolving DNA:RNA hybrids (47), how the activity of DDX5 is regulated during different cellular contexts, e.g., in response to IFNs, is presently not understood. Certainly, the regulation of DDX5 will add another level of complexity to this novel mechanism of interferon response.

Lastly, we show that in advanced HBV-related HCCs, there is absence both of DDX5 and STAT1 immunostaining (Figure 8D). Absence of STAT1 expression could provide an explanation for the lack of IFN- $\alpha$ response observed in many chronically HBV infected patients with HCC (14). Interestingly, a positive indicator for IFN- $\alpha$ responsiveness in $\mathrm{HBV}$-infected patients with HCC (14) is expression of IFIT3, a STAT1 regulated gene (13). In addition, it is presently unknown whether this mechanism of DDX5-dependent STAT1 translational regulation is also linked to the immunotherapy response of patients with HCCs. Our earlier studies identified downregulation of DDX5 to be associated with poor prognosis HBV-related HCC (16, 48), and hepatocyte reprogramming to a less-differentiated state exhibiting features of hCSCs (21), in agreement with the role of DDX5 as a barrier to pluripotency (40). Regarding immunotherapy which is based on PD-1 blockade, the PD-L1,2 ligands are induced in tumors by interferon gamma, leading to immune evasion (49). According to the mechanism described herein, we propose that absence or downregulation of DDX5 in HCCs will result in absence or 
reduced protein of STAT1, and in turn reduced PD-L1 expression, rendering these tumors refractory to PD-1 targeting immunotherapy (49).

\section{References}

1. Stark GR, Darnell JE, Jr. The JAK-STAT pathway at twenty. Immunity 2012;36:503-514.

2. Stanifer ML, Pervolaraki K, Boulant S. Differential Regulation of Type I and Type III Interferon Signaling. Int J Mol Sci 2019;20.

3. Chen K, Liu J, Liu S, Xia M, Zhang X, Han D, Jiang Y, et al. Methyltransferase SETD2-Mediated Methylation of STAT1 Is Critical for Interferon Antiviral Activity. Cell 2017;170:492-506 e414.

4. Kramer OH, Knauer SK, Greiner G, Jandt E, Reichardt S, Guhrs KH, Stauber RH, et al. A phosphorylation-acetylation switch regulates STAT1 signaling. Genes Dev 2009;23:223-235.

5. Liu S, Jiang M, Wang W, Liu W, Song X, Ma Z, Zhang S, et al. Nuclear RNF2 inhibits interferon function by promoting K33-linked STAT1 disassociation from DNA. Nat Immunol 2018;19:41-52.

6. Schneider WM, Chevillotte MD, Rice CM. Interferon-stimulated genes: a complex web of host defenses. Annu Rev Immunol 2014;32:513-545.

7. Bruix J, Qin S, Merle P, Granito A, Huang YH, Bodoky G, Pracht M, et al. Regorafenib for patients with hepatocellular carcinoma who progressed on sorafenib treatment (RESORCE): a randomised, double-blind, placebo-controlled, phase 3 trial. Lancet 2017;389:56-66.

8. Abou-Alfa GK, Meyer T, Cheng AL, El-Khoueiry AB, Rimassa L, Ryoo BY, Cicin I, et al. Cabozantinib in Patients with Advanced and Progressing Hepatocellular Carcinoma. N Engl J Med 2018;379:54-63.

9. Llovet JM, Bruix J. Molecular targeted therapies in hepatocellular carcinoma. Hepatology 2008;48:1312-1327.

10. Taniguchi T, Takaoka A. A weak signal for strong responses: interferon-alpha/beta revisited. Nat Rev Mol Cell Biol 2001;2:378-386.

11. Platanias LC. Mechanisms of type-I- and type-II-interferon-mediated signalling. Nat Rev Immunol 2005;5:375-386.

12. Lebosse F, Testoni B, Fresquet J, Facchetti F, Galmozzi E, Fournier M, Hervieu V, et al. Intrahepatic innate immune response pathways are downregulated in untreated chronic hepatitis B. J Hepatol 2017;66:897-909.

13. Xiao S, Li D, Zhu HQ, Song MG, Pan XR, Jia PM, Peng LL, et al. RIG-G as a key mediator of the antiproliferative activity of interferon-related pathways through enhancing p21 and p27 proteins. Proc Natl Acad Sci U S A 2006;103:16448-16453.

14. Yang Y, Zhou Y, Hou J, Bai C, Li Z, Fan J, Ng IOL, et al. Hepatic IFIT3 predicts interferon-alpha therapeutic response in patients of hepatocellular carcinoma. Hepatology 2017;66:152-166.

15. Zhang H, Diab A, Fan H, Mani SK, Hullinger R, Merle P, Andrisani O. PLK1 and HOTAIR Accelerate Proteasomal Degradation of SUZ12 and ZNF198 during Hepatitis B Virus-Induced Liver Carcinogenesis. Cancer Res 2015;75:2363-2374.

16. Zhang H, Xing Z, Mani SK, Bancel B, Durantel D, Zoulim F, Tran EJ, et al. RNA helicase DEAD box protein 5 regulates Polycomb repressive complex 2/Hox transcript antisense intergenic RNA function in hepatitis B virus infection and hepatocarcinogenesis. Hepatology 2016;64:1033-1048. 
17. Mani SKK, Andrisani O. Hepatitis B Virus-Associated Hepatocellular Carcinoma and Hepatic Cancer Stem Cells. Genes (Basel) 2018;9.

18. Margueron R, Reinberg $\mathrm{D}$. The Polycomb complex PRC2 and its mark in life. Nature 2011;469:343-349.

19. Jarmoskaite I, Russell R. RNA helicase proteins as chaperones and remodelers. Annu Rev Biochem 2014;83:697-725.

20. Jankowsky E, Fairman ME. RNA helicases--one fold for many functions. Curr Opin Struct Biol 2007;17:316-324.

21. Mani SKK, Yan B, Cui Z, Sun J, Utturkar S, Foca A, Fares N, et al. Restoration of RNA helicase DDX5 suppresses hepatitis B virus (HBV) biosynthesis and Wnt signaling in HBV-related hepatocellular carcinoma. Theranostics 2020;10:10957-10972.

22. Bugaut A, Balasubramanian S. 5'-UTR RNA G-quadruplexes: translation regulation and targeting. Nucleic Acids Res 2012;40:4727-4741.

23. Yang D. G-Quadruplex DNA and RNA. Methods Mol Biol 2019;2035:1-24.

24. Kumari S, Bugaut A, Huppert JL, Balasubramanian S. An RNA G-quadruplex in the 5' UTR of the NRAS proto-oncogene modulates translation. Nat Chem Biol 2007;3:218-221.

25. Kwok CK, Marsico G, Sahakyan AB, Chambers VS, Balasubramanian S. rG4-seq reveals widespread formation of G-quadruplex structures in the human transcriptome. Nat Methods 2016;13:841-844.

26. Wu G, Xing Z, Tran EJ, Yang D. DDX5 helicase resolves G-quadruplex and is involved in MYC gene transcriptional activation. Proc Natl Acad Sci U S A 2019;116:20453-20461.

27. Lucifora J, Durantel D, Belloni L, Barraud L, Villet S, Vincent IE, Margeridon-Thermet S, et al. Initiation of hepatitis B virus genome replication and production of infectious virus following delivery in HepG2 cells by novel recombinant baculovirus vector. J Gen Virol 2008;89:1819-1828.

28. Ladner SK, Otto MJ, Barker CS, Zaifert K, Wang GH, Guo JT, Seeger C, et al. Inducible expression of human hepatitis B virus (HBV) in stably transfected hepatoblastoma cells: a novel system for screening potential inhibitors of HBV replication. Antimicrob Agents Chemother 1997;41:1715-1720.

29. Qiu Z, Li H, Zhang Z, Zhu Z, He S, Wang X, Wang P, et al. A Pharmacogenomic Landscape in Human Liver Cancers. Cancer Cell 2019;36:179-193 e111.

30. Ribeiro de Almeida C, Dhir S, Dhir A, Moghaddam AE, Sattentau Q, Meinhart A, Proudfoot NJ. RNA Helicase DDX1 Converts RNA G-Quadruplex Structures into R-Loops to Promote IgH Class Switch Recombination. Mol Cell 2018;70:650-662 e658.

31. Hatzakis E, Okamoto K, Yang D. Thermodynamic stability and folding kinetics of the major Gquadruplex and its loop isomers formed in the nuclease hypersensitive element in the human c-Myc promoter: effect of loops and flanking segments on the stability of parallel-stranded intramolecular Gquadruplexes. Biochemistry 2010;49:9152-9160.

32. Yang L, Yang JL, Byrne S, Pan J, Church GM. CRISPR/Cas9-Directed Genome Editing of Cultured Cells. Curr Protoc Mol Biol 2014;107:31 31 31-17.

33. Zhu X, Xu Y, Yu S, Lu L, Ding M, Cheng J, Song G, et al. An efficient genotyping method for genome-modified animals and human cells generated with CRISPR/Cas9 system. Sci Rep 2014;4:6420.

34. Han FX, Wheelhouse RT, Hurley LH. Interactions of TMPyP4 and TMPyP2 with Quadruplex DNA. Structural Basis for the Differential Effects on Telomerase Inhibition. Journal of the American Chemical Society 1999;121:3561-3570.

35. Kumari S, Bugaut A, Balasubramanian S. Position and stability are determining factors for translation repression by an RNA G-quadruplex-forming sequence within the 5' UTR of the NRAS protooncogene. Biochemistry 2008;47:12664-12669.

36. Lander ES. The Heroes of CRISPR. Cell 2016;164:18-28. 
37. Del Villar-Guerra R, Gray RD, Chaires JB. Characterization of Quadruplex DNA Structure by Circular Dichroism. Curr Protoc Nucleic Acid Chem 2017;68:17 18 11-17 1816.

38. Zhang J, Lau MW, Ferré-D'Amaré AR. Ribozymes and Riboswitches: Modulation of RNA Function by Small Molecules. Biochemistry 2010;49:9123-9131.

39. Linder P, Jankowsky E. From unwinding to clamping - the DEAD box RNA helicase family. Nat Rev Mol Cell Biol 2011;12:505-516.

40. Li H, Lai P, Jia J, Song Y, Xia Q, Huang K, He N, et al. RNA Helicase DDX5 Inhibits Reprogramming to Pluripotency by miRNA-Based Repression of RYBP and its PRC1-Dependent and -Independent Functions. Cell Stem Cell 2017;20:571.

41. Guo L, Lin L, Wang X, Gao M, Cao S, Mai Y, Wu F, et al. Resolving Cell Fate Decisions during Somatic Cell Reprogramming by Single-Cell RNA-Seq. Mol Cell 2019;73:815-829 e817.

42. Guo YL, Carmichael GG, Wang R, Hong X, Acharya D, Huang F, Bai F. Attenuated Innate Immunity in Embryonic Stem Cells and Its Implications in Developmental Biology and Regenerative Medicine. Stem Cells 2015;33:3165-3173.

43. Millevoi S, Moine H, Vagner S. G-quadruplexes in RNA biology. Wiley Interdiscip Rev RNA 2012;3:495-507.

44. Qin Y, Hurley LH. Structures, folding patterns, and functions of intramolecular DNA Gquadruplexes found in eukaryotic promoter regions. Biochimie 2008;90:1149-1171.

45. Arora A, Dutkiewicz M, Scaria V, Hariharan M, Maiti S, Kurreck J. Inhibition of translation in living eukaryotic cells by an RNA G-quadruplex motif. RNA 2008;14:1290-1296.

46. Nie J, Jiang $M$, Zhang $X$, Tang $H$, Jin $H$, Huang $X$, Yuan $B$, et al. Post-transcriptional Regulation of Nkx2-5 by RHAU in Heart Development. Cell Rep 2015;13:723-732.

47. Mersaoui SY, Yu Z, Coulombe Y, Karam M, Busatto FF, Masson JY, Richard S. Arginine methylation of the DDX5 helicase RGG/RG motif by PRMT5 regulates resolution of RNA:DNA hybrids. EMBO J 2019;38:e100986.

48. Zhang $\mathrm{H}$, Zhang $\mathrm{Y}$, Zhu $\mathrm{X}$, Chen $\mathrm{C}$, Zhang $\mathrm{C}$, Xia $\mathrm{Y}$, Zhao $\mathrm{Y}$, et al. DEAD Box Protein 5 Inhibits Liver Tumorigenesis by Stimulating Autophagy via Interaction with p62/SQSTM1. Hepatology 2018.

49. Garcia-Diaz A, Shin DS, Moreno BH, Saco J, Escuin-Ordinas H, Rodriguez GA, Zaretsky JM, et al. Interferon Receptor Signaling Pathways Regulating PD-L1 and PD-L2 Expression. Cell Rep 2017;19:11891201. 
bioRxiv preprint doi: https://doi.org/10.1101/2020.09.25.313684; this version posted September 25, 2020. The copyright holder for this preprint (which was not certified by peer review) is the author/funder. All rights reserved. No reuse allowed without permission. 

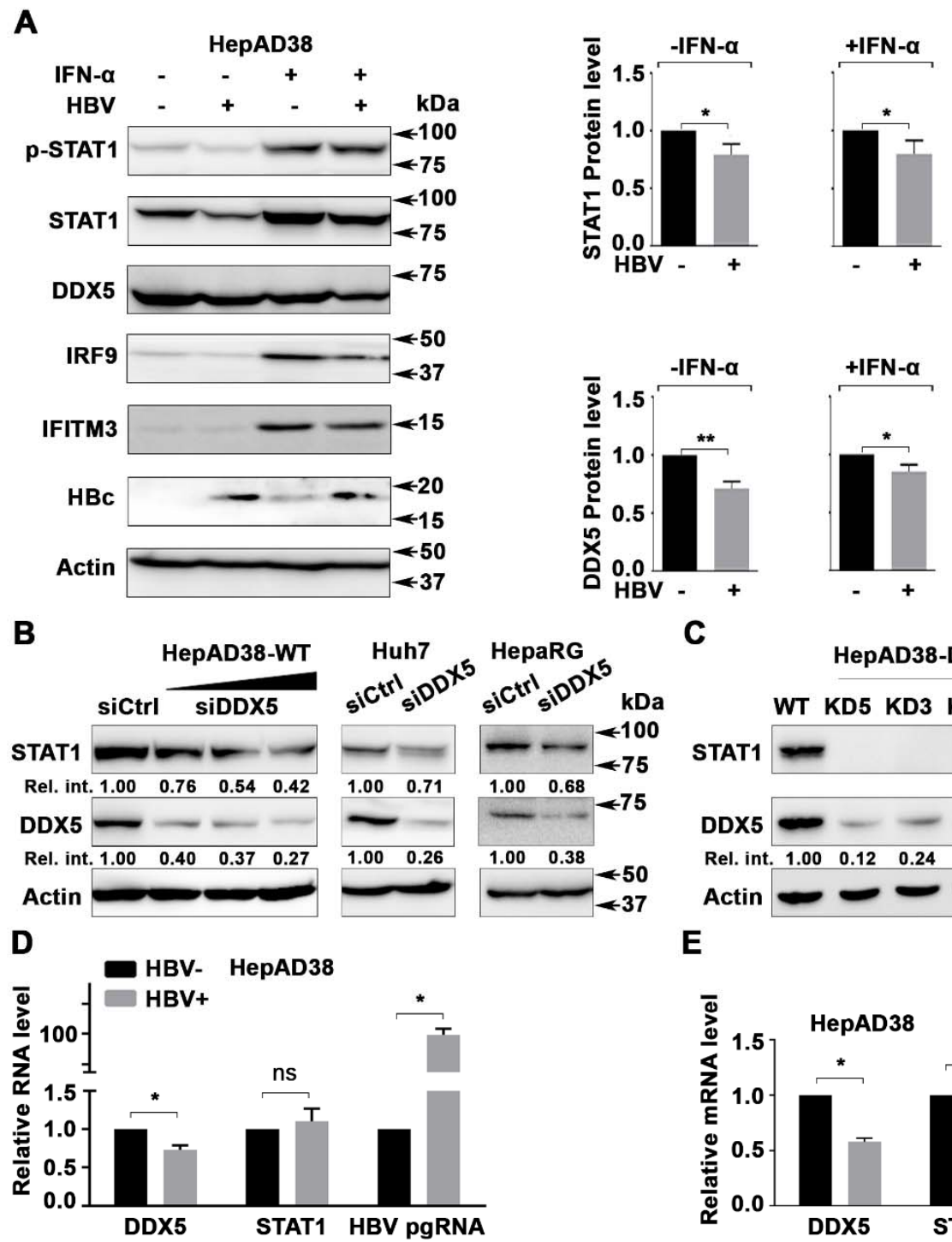

F

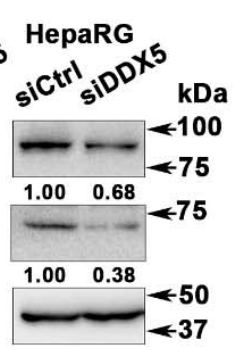

C

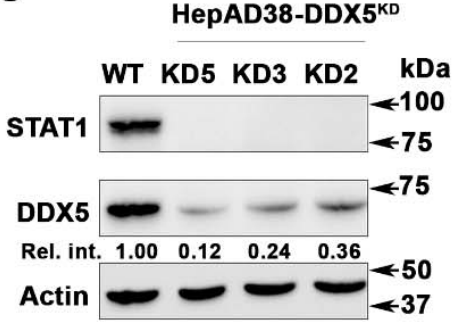

E
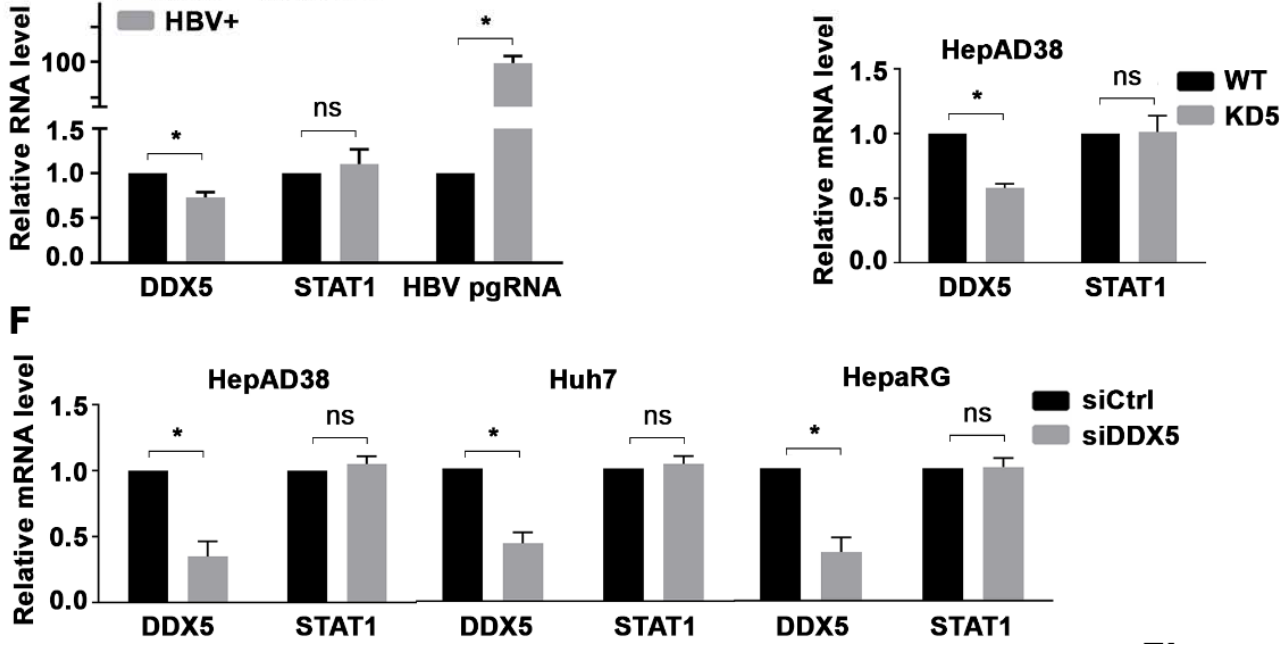

Figure 1. DDX5 knockdown regulates STAT1 mRNA translation. (A) Immunoblots of IFN-

$\alpha$ induced proteins using lysates from HepAD38 cells with (+) or without (-) HBV replication for 5 days as a function of IFN- $\alpha(500 \mathrm{ng} / \mathrm{ml})$ treatment for the last $24 \mathrm{~h}$. (Right panel) quantification of DDX5 and STAT1 protein level, by ImageJ software, from three independent biological 
replicates. *: $\mathrm{p}<0.05, * *: \mathrm{p}<0.01$; Error bars indicate Mean \pm SEM. Immunoblot of indicated proteins in: (B) HepAD38, Huh7 and HepaRG cells transfected with DDX5 siRNA (siDDX5) or negative control siRNA (siCtrl) for $48 \mathrm{~h}$, and (C) in WT and DDX5 knockdown HepAD38 cell lines KD2, KD3 and KD5. (D)-(F) qRT-PCR of HBV pgRNA and STAT1 mRNA using RNA from (D) HepAD38 cells with (+) or without (-) HBV replication for 5 days; (E) WT and KD5 HepAD38 cells, and (F) HepAD38, Huh7, and HepaRG cells transfected with siDDX5 or siCtrl for 48h. Statistical analysis of DDX5 and STAT1 mRNA levels from three biological replicates. *: p<0.05, ns: not significant; Error bars indicate Mean \pm SEM. 

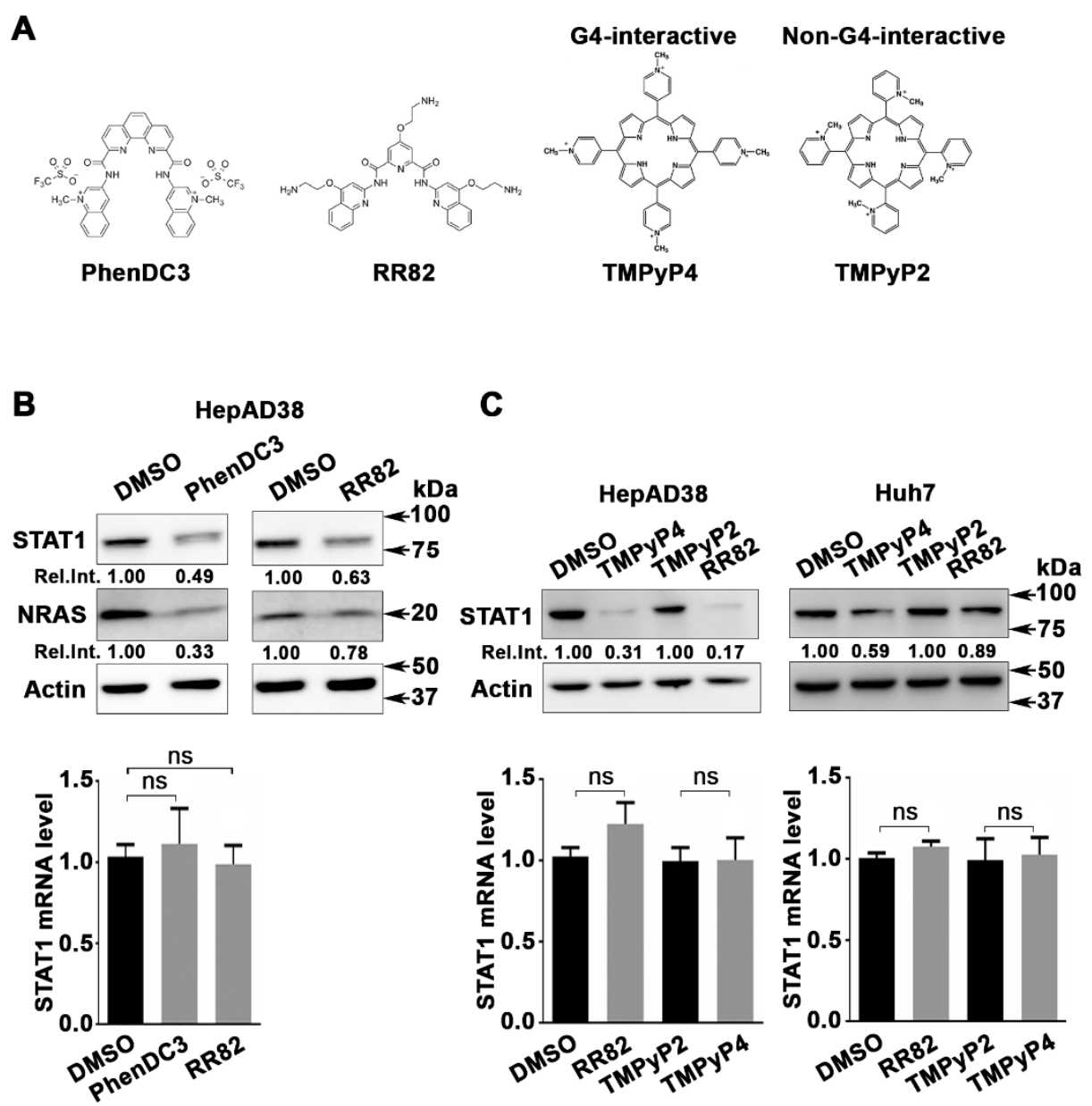

Figure 2. G-quadruplex stabilizing drugs reduce STAT1 protein levels. (A) Chemical structure of G4 stabilizing compounds PhenDC3, RR82, TMPyP4 and TMPyP2. (B) Immunoblots of STAT1 and NRAS, using lysates from HepAD38 cells treated for $48 \mathrm{~h}$ with PhenDC3 $(5 \mu \mathrm{M})$ or RR82 $(5 \mu \mathrm{M})$. (Lower panel) qRT-PCR of STAT1 mRNA using RNA from HepAD38 cells treated with DMSO, PhenDC3 $(5 \mu \mathrm{M})$ or RR82 $(5 \mu \mathrm{M})$ for $48 \mathrm{~h}$. (C) Immunoblots of STAT1 from lysates of HepAD38 and Huh7 cells treated with TMPyP4 (5 $\mu \mathrm{M})$ or TMPyP2 $(5 \mu \mathrm{M})$ for $48 \mathrm{~h}$. (Lower panel), qRT-PCR of STAT1 mRNA from HepAD38 and Huh7 cells, treated as indicated. Statistical analysis of STAT1 mRNA is from three biological replicates. ns: not significant; Error bars indicate Mean \pm SEM. 
A
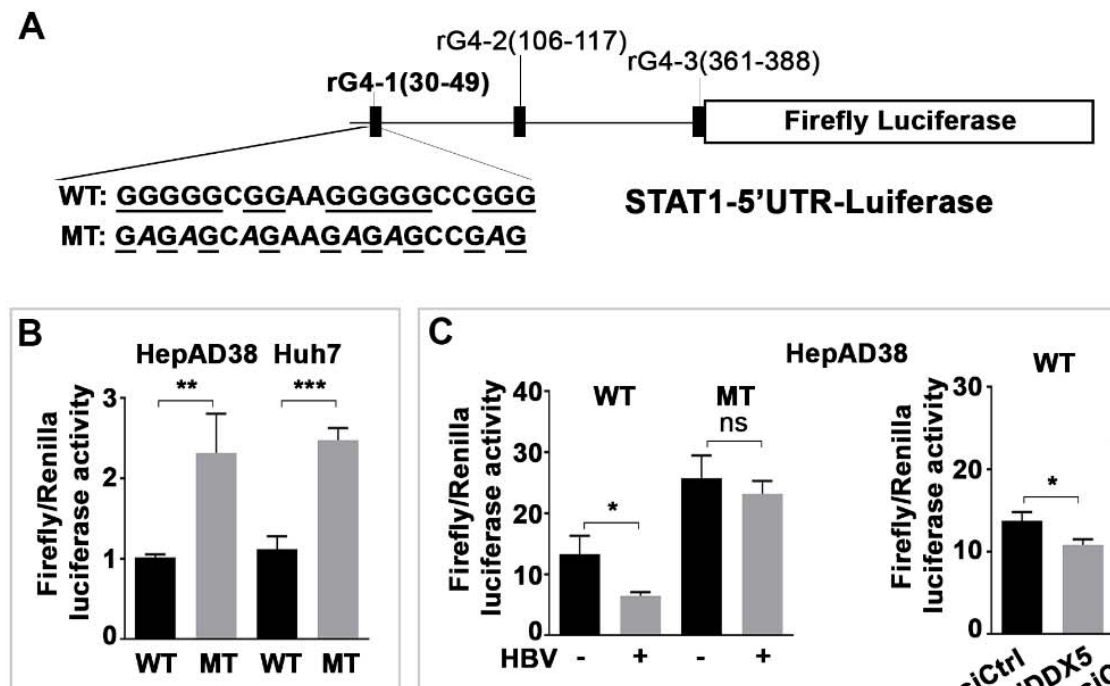

HepAD38
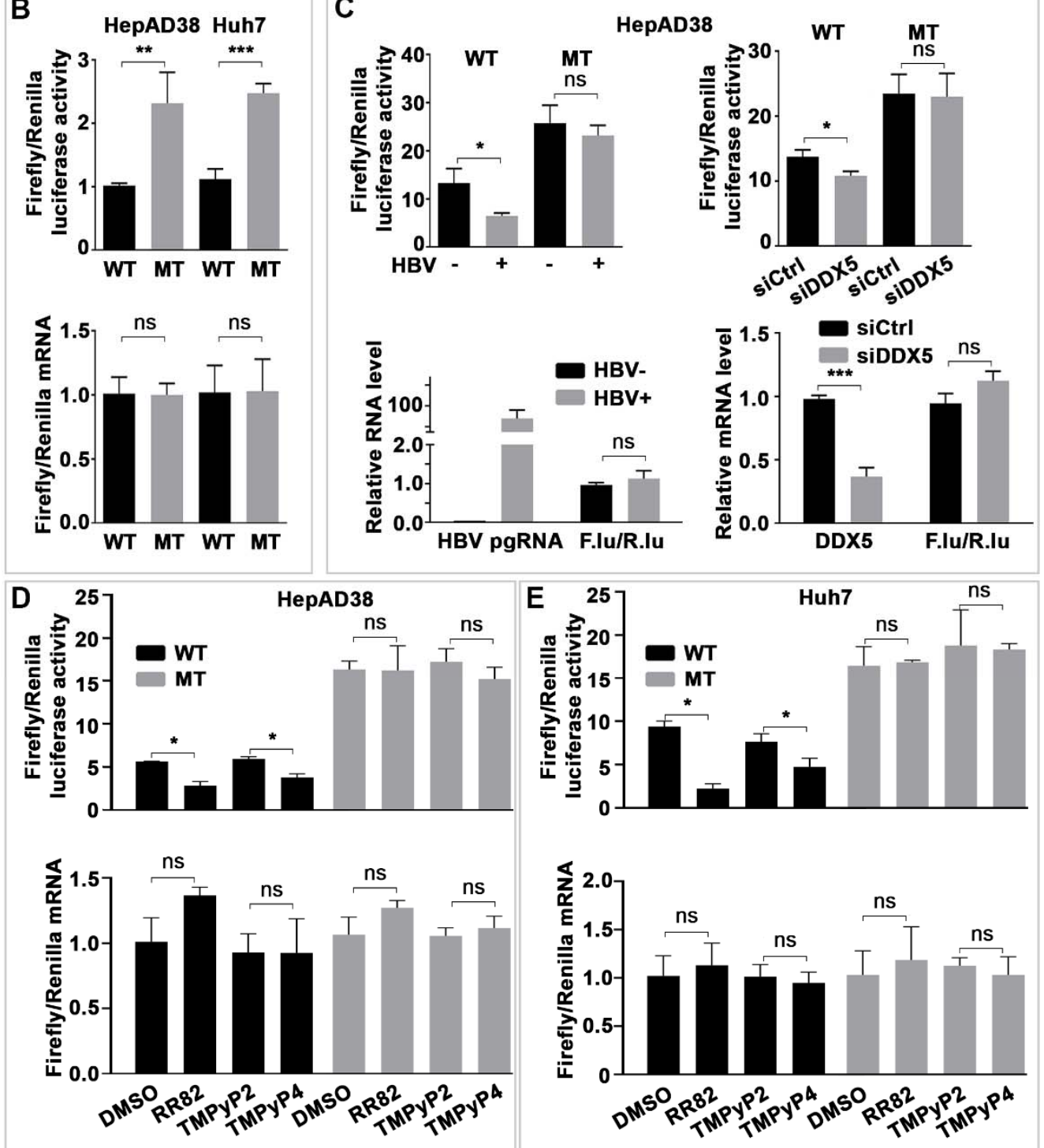

Figure 3. G-quadruplex (rG4) regulates STAT1 expression post-transcriptionally. (A)

Human STAT1 5'UTR upstream of Firefly (F.) Luciferase reporter, driven from SV40 promoter.

Putative rG4 sequences in 5' UTR indicated as rG4-1, rG4-2 and rG4-3. WT rG4-1 nucleotide sequence is shown. Italics indicate site-directed changes in mutant MT-rG4-1. (B) - (E) Ratio of 
Firefly/Renilla luciferase activity at $24 \mathrm{~h}$ after co-transfection of WT or MT STAT1-5'UTR-F. Luciferase and Renilla-Luciferase expression plasmids. Lower panels, ratio of Firefly/Renilla luciferase mRNAs quantified by qRT-PCR. (B) HepAD38 and Huh7 cells. (C) HepAD38 cells with (+) or without (-) HBV replication for 5 days. (D) HepAD38 cells, and (E) Huh7 cells treated with indicated G-quadruplex stabilizing drugs $(5 \mu \mathrm{M})$ for $24 \mathrm{~h}$. Statistical analysis from three independent biological replicates. *: $\mathrm{p}<0.05, * *: \mathrm{p}<0.01, * * *: \mathrm{p}<0.001$, ns: not significant; Error bars indicate Mean \pm SEM. 
A

Huh7 CRISPR edited clones

WT rG4-1: 5 ',..CCCGGTGATTGGTGGGGGGGAAGGGGCCGGG...3'

C17 rG4-1, allele 1: 5'...CCCGGTGATT...............GGCGG..........GGGCGGG....

C17 rG4-1, allele 2: 5'...CCCGGTGATT.........GGGGGGGAAGGGGGCCGGG...3'

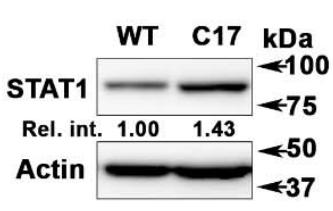

B

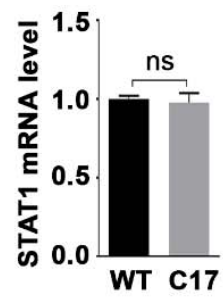

HepaRG CRISPR edited clones

WT rG4-1: $5^{\prime}, \ldots$ CCCGGTGATTGGTGGGGGGGAAGGGGCCGGG...3'

.CGGAAGGGGGCCGGG...3'

C5 rG4-1, allele 2: $5^{\prime}$....CCC.

GGGGCCGGG...3'

C9 rG4-1, allele 1: 5'...CCCGGTGATT.........GGGGGCGGAAGGGGGCGGG....3'

C9 rG4-1, allele 2: 5'...CCCGGTGATTGGT.

..GGGGCCGGG...3'
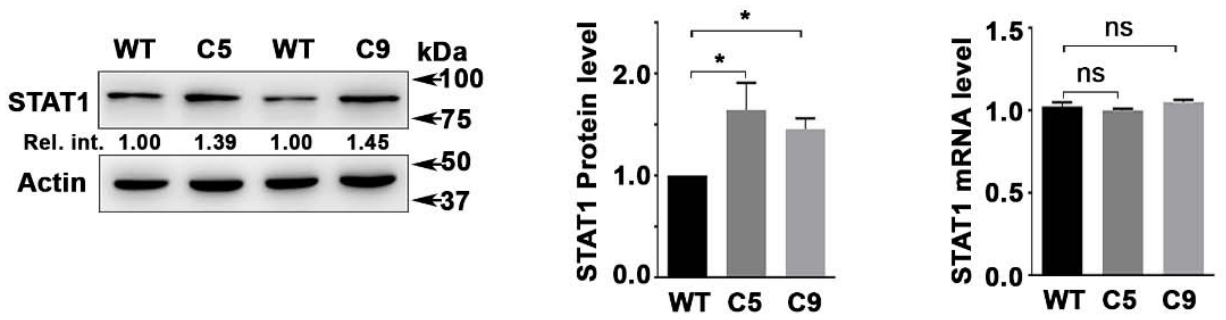

Figure 4. Genomic editing of rG4-1 increases STAT1 protein levels. (A) Sequence of CRIPSR/Cas9 edited rG4-1 sequence in (A) Huh7 and (B) HepaRG cells. Immunoblot of STAT1 in indicated cell lines. Right panels, quantification of STAT1 protein by ImageJ software, and qRT-PCR of STAT1 mRNA, from three independent biological replicates. *: p<0.05, ns: not significant; Error bars indicate Mean \pm SEM. 
A
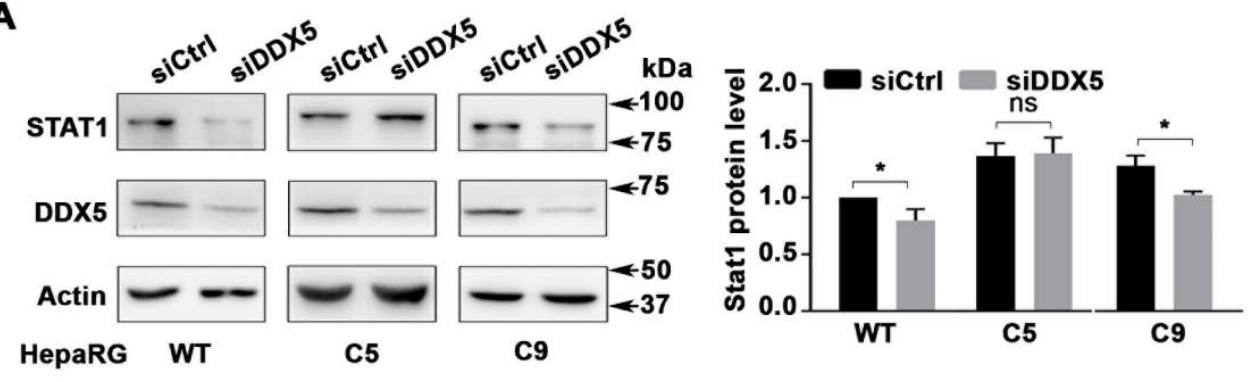

B

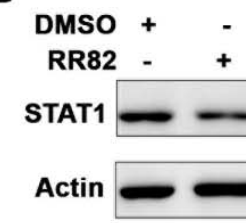

HepaRG WT

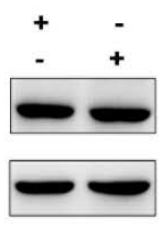

C5

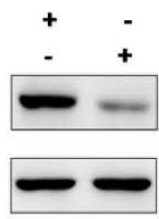

C9

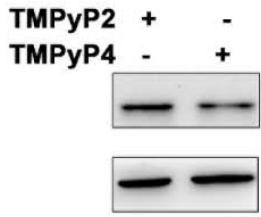

WT

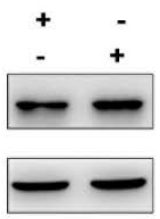

C5

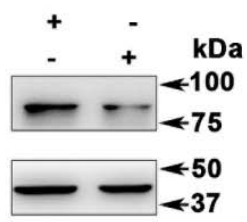

C9
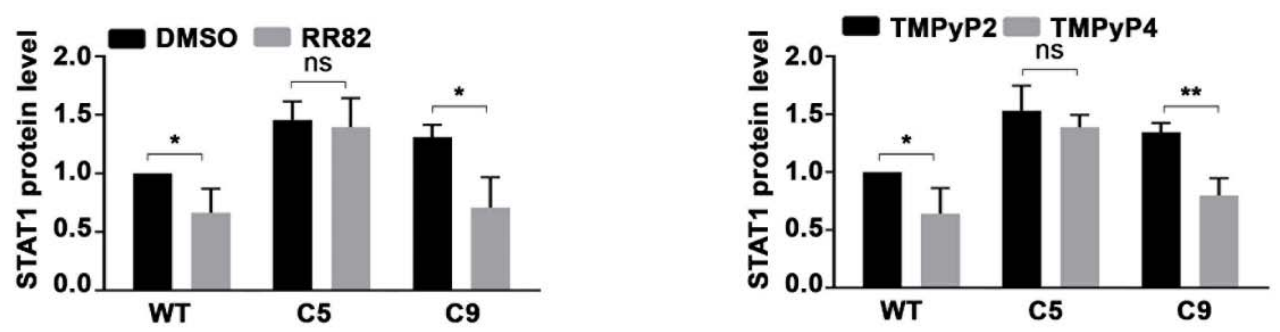

C
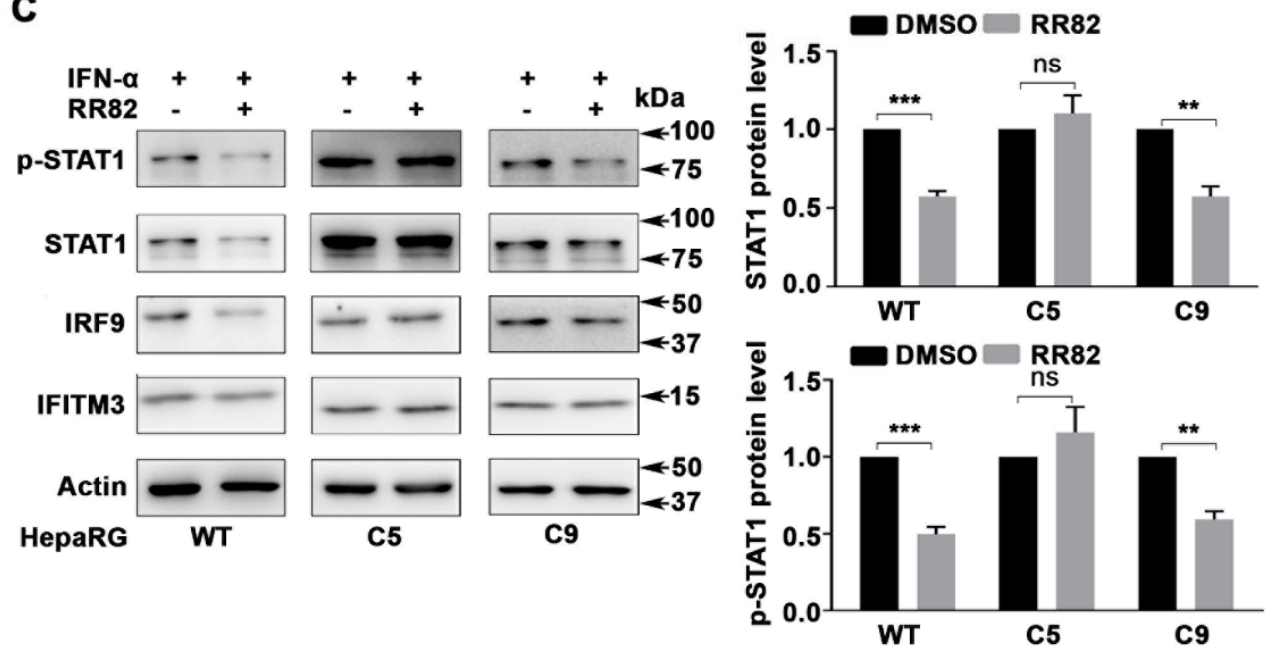

Figure 5. Effect of G-quadruplex stabilizing drugs on rG4-1 edited HepaRG cells. Immunoblots of indicated proteins using lysates from indicated HepaRG cells (WT, C5, and C9).

(A) After transfection of siCtrl or siDDX5 RNA for $48 \mathrm{~h}$; (B) following treatment with RR82 (5 $\mu \mathrm{M})$, TMPyP4 (5 $\mu \mathrm{M})$ or TMPyP2 $(5 \mu \mathrm{M})$ for $48 \mathrm{~h}$, and (C) treatment with RR82 (5 $\mu \mathrm{M})$ for 48 $\mathrm{h}$ in combination with IFN- $\alpha(500 \mathrm{ng} / \mathrm{ml})$ for the last $24 \mathrm{~h}$. Quantification (A-C) from three 
bioRxiv preprint doi: https://doi.org/10.1101/2020.09.25.313684; this version posted September 25, 2020. The copyright holder for this preprint (which was not certified by peer review) is the author/funder. All rights reserved. No reuse allowed without permission.

independent biological replicates. $*: \mathrm{p}<0.05,{ }^{*}: \mathrm{p}<0.01$, ***: $\mathrm{p}<0.001$, ns: not significant. Error bars indicate Mean \pm SEM. 
A WT RNA: 5'CGgUGAUUGGUGGGGGGAAGGGGGCCGGGCGC3'

C5-1 RNA: 5'UUUUUC.. CGGAAGGGGGCCGGGCGCUUUUU3'

C5-2 RNA: 5'UUUUUC ..GGGGCCGGGCGCUUUUU3' C9-1 RNA: 5'CGGUGAUU.....GGGGGCGGAAGGGGCCGGGCGC3'

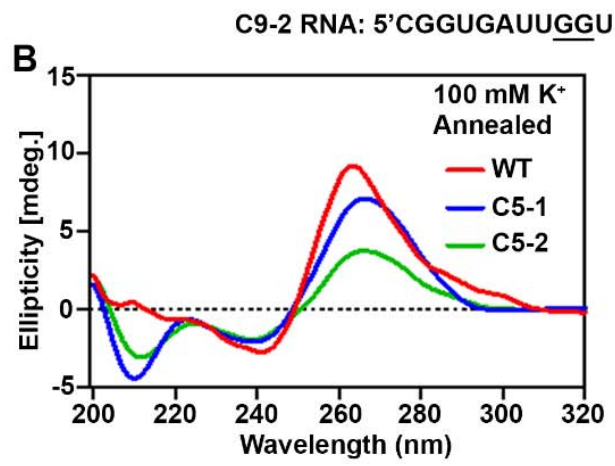
..GGGGCCGGGCGC3'
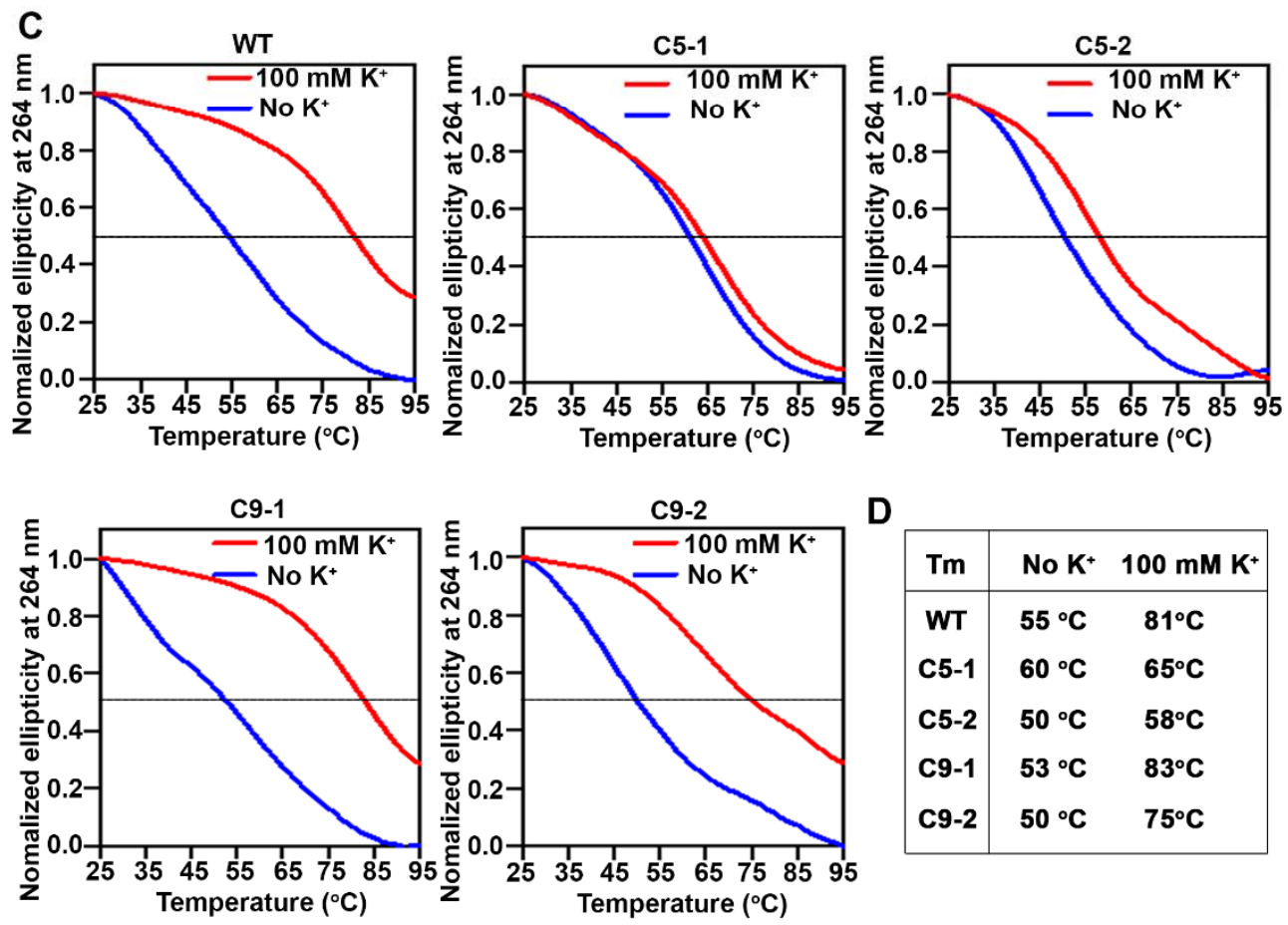

Figure 6. The rG4-1 sequence in 5'UTR of STAT1 mRNA forms G-quadruplex. (A)

Synthetic RNA oligonucleotides of WT STAT1 rG4-1 and corresponding mutants in HepaRG clones C5 and C9. (B) CD spectroscopy measurement, and (C) melting curves of RNA oligonucleotides annealed by heating to $95^{\circ} \mathrm{C}$ and slowly cooled down to room temperature. (D) Melting temperature $(\mathrm{Tm})$, without and with $(100 \mathrm{mM}) \mathrm{KCl}$, calculated from A-C. 


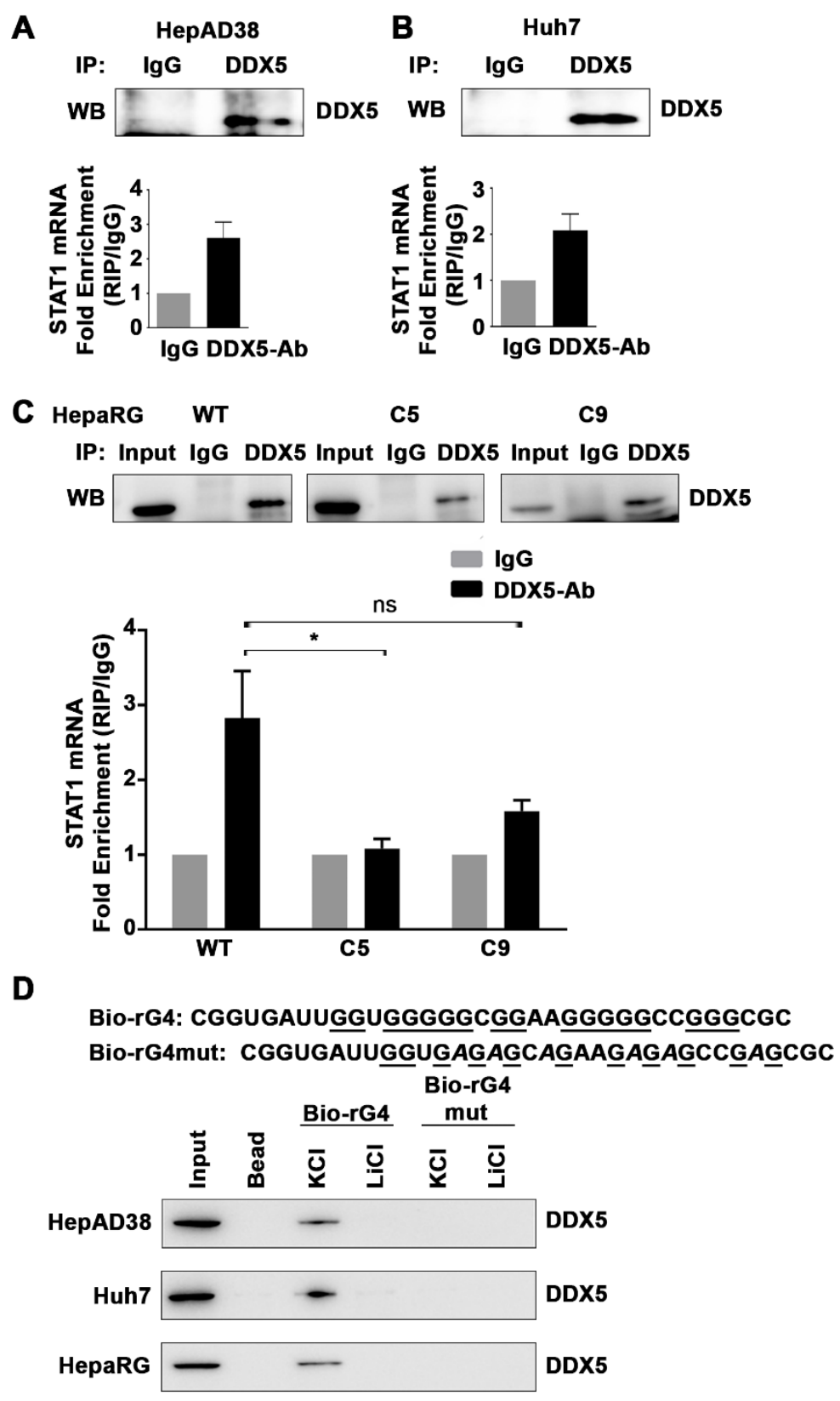

Figure 7. DDX5 binds STAT1 mRNA. Ribonucleoprotein immunoprecipitation (RIP) assays with DDX5 antibody performed in (A) HepAD38, (B) Huh7, and (C) HepaRG cells, WT, C5 and C9. DDX5 enriched RNAs quantified by qRT-PCR using STAT1 primers. Results are from three biological replicates. *: $\mathrm{p}<0.05$, ns=not significant. (D) RNA pulldown assays using synthetic, biotinylated RNA oligonucleotides, WT and mutated rG4-1, in $100 \mathrm{mM} \mathrm{KCl}$ or 100 
bioRxiv preprint doi: https://doi.org/10.1101/2020.09.25.313684; this version posted September 25, 2020. The copyright holder for this preprint (which was not certified by peer review) is the author/funder. All rights reserved. No reuse allowed without permission.

$\mathrm{mM} \mathrm{LiCl}$, bound to lysates from indicated cell lines, followed by immunoblots with DDX5 antibody. A representative assay is shown from three independent experiments. 

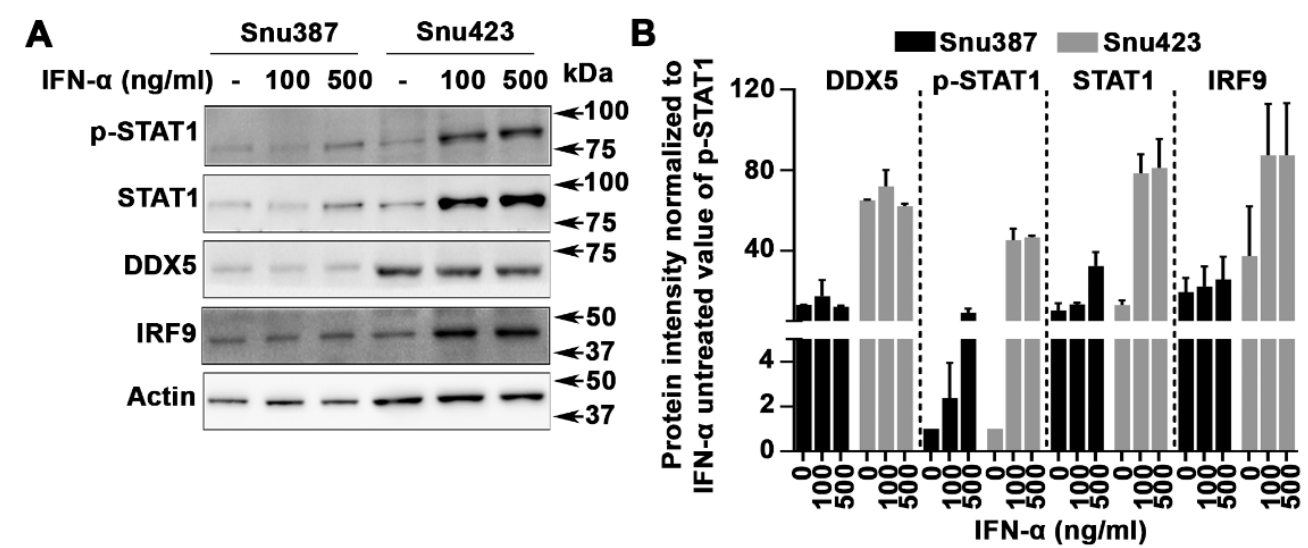

C
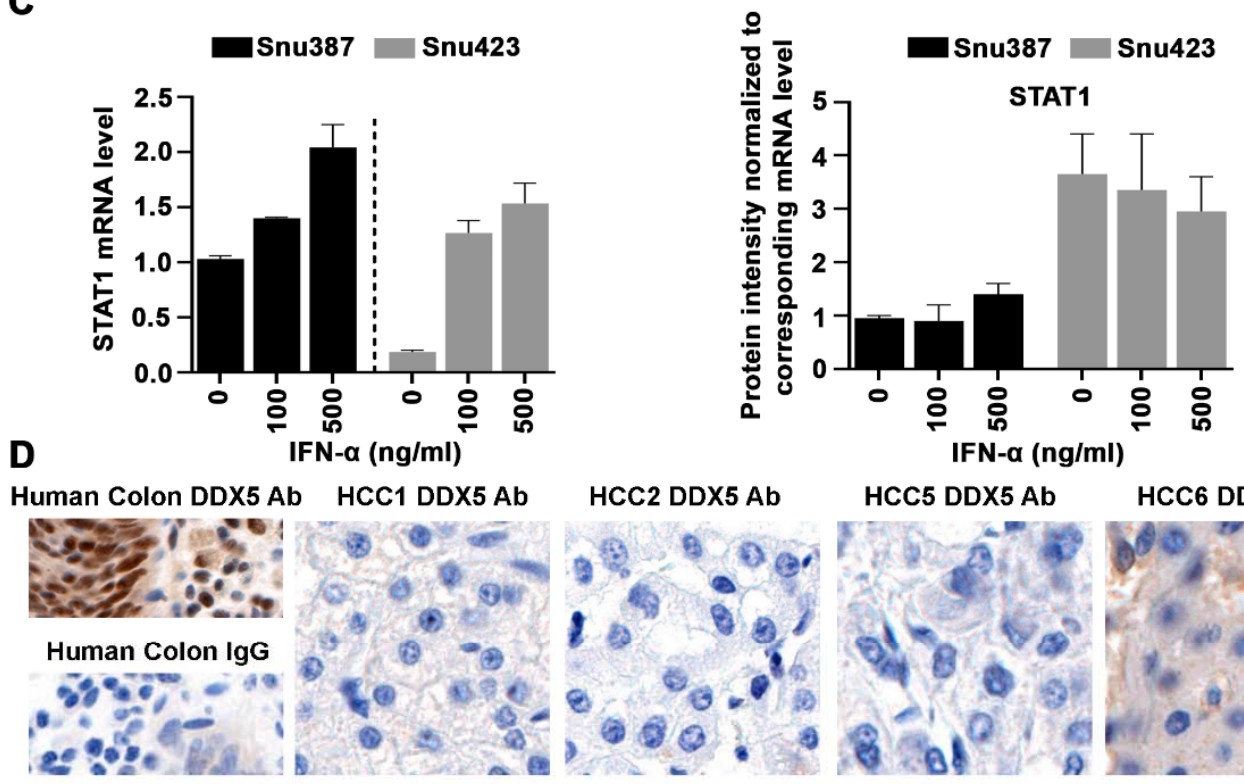

Human Colon STAT1 Ab HCC1 STAT1 Ab

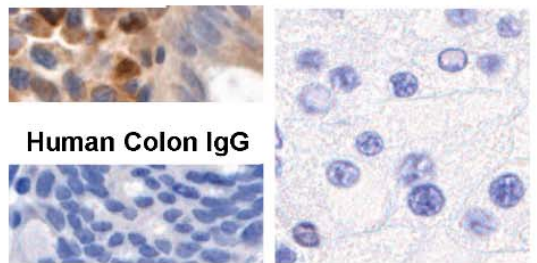

HCC2 STAT1 Ab

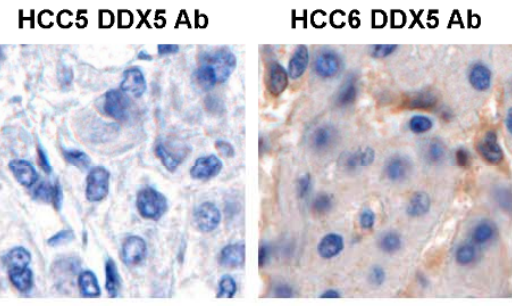

\begin{tabular}{|ccccc|}
\hline Case No. & HCC1 & HCC2 & HCC5 & HCC6 \\
\hline Edmonson's grade & 3 & $2 / 3$ & 3 & 3 \\
\hline
\end{tabular}

Figure 8. DDX5 expression level vs. magnitude of interferon response. (A) Immunoblots using lysates from indicated cell lines treated with IFN- $\alpha$ (100 and $500 \mathrm{ng} / \mathrm{ml})$ for $12 \mathrm{~h}$. (B) Quantification shows ratio of p-STAT1, STAT1, IRF9 and DDX5 relative to level of p-STAT1 in IFN- $\alpha$ untreated cells. Results are average from three independent experiments. (C)(Left 
panel) Quantification by RT qPCR of STAT1 mRNA in Snu387 and 423 cell lines treated with IFN- $\alpha$ as indicated for $12 \mathrm{~h}$. (Right panel) STAT1 protein from (A) normalized to STAT1 mRNA in Snu387 vs. Snu423 cell lines, under the indicated treatment. (D) Immunohistrochemistry of HBV-related HCCs and normal human colon tissue, used as positive control, with DDX5 and STAT1 antibodies vs. IgG, performed as described (16). 